\title{
Proof of Concept: Matrix metalloproteinase inhibitor decreases inflammation and improves muscle insulin sensitivity in people with type 2 diabetes
}

\author{
Karen Frankwich ${ }^{2}$, Courtney Tibble 2 , Moises Torres-Gonzalez ${ }^{2}$, Mariah Bonner ${ }^{1,5}$, Roy Lefkowitz ${ }^{3,4}$, Matt Tyndall ${ }^{3}$,
} Geert W Schmid-Schönbein ${ }^{3}$, Francisco Villarreal ${ }^{2}$, Mike Heller ${ }^{3}$ and Karen Herbst ${ }^{1,2^{*}}$

\begin{abstract}
Background: Obesity is a state of subclinical inflammation resulting in loss of function of insulin receptors and decreased insulin sensitivity. Inhibition of the inflammatory enzymes, matrix metalloproteinases (MMPs), for 6 months in rodent models restores insulin receptor function and insulin sensitivity.

Methods: This 12-week double-blind, randomized, placebo (PL)-controlled proof-of-concept study was performed to determine if the MMP inhibitor (MMPI), doxycycline, decreased global markers of inflammation and enhanced muscle insulin sensitivity in obese people with type 2 diabetes (DM2). The study included non-DM2 controls $(n=15)$, and DM2 subjects randomized to PL $(n=13)$ or doxycycline $100 \mathrm{mg}$ twice daily (MMPI; $n=11)$. All participants were evaluated on Day 1; MMPI and PL groups were also evaluated after 84 days of treatment.
\end{abstract}

Results: There was a significant decrease in inflammatory markers $C$-reactive protein $(P<0.05)$ and myeloperoxidase $(P=0.01)$ in the MMPI but not PL group. The MMPI also significantly increased skeletal muscle activated/total insulin signaling mediators: 3'phosphoinositide kinase-1 (PDK1) $(p<0.03)$, protein kinase B (PKB/Akt) $(p<0.004)$, and glycogen synthase kinase $3 ß$ (GSK3ß) $(p<0.03)$.

Conclusions: This study demonstrated short term treatment of people with diabetes with an MMPI resulted in decreased inflammation and improved insulin sensitivity. Larger, longer studies are warranted to determine if doxycycline can improve glucose control in people with diabetes.

Trial Registration: Clinicaltrials.gov NCT01375491

Keywords: Diabetes, Doxycycline, Insulin sensitivity, Matrix metalloproteinases, Myeloperoxidase

\section{Background}

As early as 1993, obesity was recognized as a heightened state of inflammation with inflammatory cells increasing within enlarging adipose tissue depots [1]. As part of inflammation, expression levels and activity of matrix metalloproteinases (MMPs), a family of extracellular endopeptidases, are significantly elevated in rodent

\footnotetext{
* Correspondence: Karen.herbst@va.gov

'Veteran's Affairs, San Diego Healthcare System, 3350 La Jolla Village Drive (111 G), San Diego, CA 92161, USA

2Department of Medicine, Division of Endocrinology \& Metabolism, University of California, 9500 Gilman Drive, \#0673 La Jolla, San Diego, CA 92093, USA

Full list of author information is available at the end of the article
}

models of obesity [2] and obese humans. [3-5] MMPs, ubiquitously found in tissue and secreted by immune cells, cleave extra-cellular matrix components of body tissues and other proteins during wound repair, tissue remodeling, cancer growth and metastases, and inflammation. MMPs reported to be elevated in obesity compared to healthy control populations include MMP-2, $-3,-8$ and -9 , with less elevation of MMP-7 [3] [6], the latter thought to be a marker of adipocyte maturity [7].

In animal models of diabetes and hypertension, excessive proteolytic MMP activity cleaves the extracellular domain of the insulin receptor, as well as other receptors, resulting in loss of function, including a reduction 
in insulin sensitivity. [8,9] Blockade of proteases by an MMP inhibitor (MMPI) therefore improved not only MMP levels and activity but blood glucose levels by $33 \%$ in mice. [9] Regulation of MMPs is, however, complex, where transcriptional regulators and tissue inhibitors of MMP (TIMPs) modulate MMP levels and activity, respectively. Only examining MMP levels may therefore, not provide complete information. For example, MMP-2 and MMP-9 decreased after exercise-induced weight loss $[10,11]$ but in a separate study, MMP-2 and MMP-3 levels did not decline after bariatric surgery. [7] Clinical outcome studies are therefore warranted to determine whether MMP inhibition reduces inflammation and improves insulin sensitivity in obese diabetic patients.

Doxycycline, a synthetic derivative of tetracycline, is a broad spectrum inhibitor of MMP-1, -2, -3, -8, -9, -10, and -13 [9,12-15]. Doxycycline has been used successfully as an MMPI in a number of pathological states in which MMPs are known to be elevated. For example, doxycycline significantly reduced progressive aortic degeneration after aneurysm repair along with MMP-9 levels in human subjects after 6 months of treatment [16]. Doxycycline was also more effective than a betablocker at preventing thoracic aortic aneurysm development in Marfan syndrome through inhibition of MMP-2 and -9 [15]. Golub et al. also demonstrated inhibition of MMP activity in rodent models of DM2 by MMPIs of which doxycycline was the most potent. [14,17]

A means to determine if MMPI translates into improved insulin sensitivity in diabetic patients is to assess the activity of molecules in the insulin signaling pathway in skeletal muscle. Insulin binding to its receptor activates phosphatidyl inositol-3-kinase (PI3K) resulting in recruitment and phosphorylation of PDK1 (pPDK1), which in turn phosphorylates and activates Akt (pAkt). [18] pAkt triggers an increase in insulin responsive glucose transporter (GLUT4) translocation and recruitment to the cell membrane, increasing glucose uptake by the cell. pAkt also phosphorylates and thus inactivates GSK3 $\beta$ (pGSK3 $\beta$ ). This prevents GSK3 $\beta$ from phosphorylating (and thus inactivating) glycogen synthase - which leaves the glycogen synthase active to continue glycogen production. Enhancement of insulin sensitivity in muscle, therefore, should increase levels of pPDK1, pAkt and pGSK3 $\beta$.

In a proof of concept clinical trial we determined whether doxycycline (MMPI) can reduce inflammation and improve insulin sensitivity. For this purpose, we enrolled DM2 patients into a double-blind randomized, short-term, clinical trial to receive 84 days of either MMPI or PL. Markers of inflammation and muscle insulin signaling mediators were measured before and after treatment and compared to placebo controls.

\section{Methods}

\section{Eligibility and exclusion criteria}

Participants were eligible if they had DM2 $<10$ years with A1c $7.5-10 \%$, no or stable retinopathy, and normal creatinine. Because this was a proof of concept study, participants could use any hypoglycemic medication including insulin but not thiazolidinediones, which are known to inhibit MMPs [19]. Using published data on relative MMP-9 levels [20], we estimated a power greater than $99 \%$ at a two-sided alpha of $5 \%$ to see a difference in mean C-reactive protein (CRP) and MMP levels between DM groups with 15 subjects per group.

\section{Study design}

This study was approved by the University of California, San Diego (UCSD) Human Research Protection Program and the Research and Development Committee at the Veterans Affairs San Diego Healthcare System which support research that is in compliance with the Helsinki Declaration as part of the United States' Office for Human Research Protections. All participants completed an informed consent process prior to enrollment. The study was a double-blind, randomized, PL-controlled trial of the oral antibiotic, doxycycline, compared to PL in obese patients with DM2. The participants with DM2 were randomly assigned to either $100 \mathrm{mg}$ doxycycline (MMPI) twice daily or PL until day 84 (D84). The main inflammatory outcome measures, CRP and myeloperoxidase (MPO) levels, were measured at baseline (day 1; D1) and D84. Muscle biopsies for total and phosphorylated PDK1, Akt, and GSK3 $\beta$ were obtained for 7 consecutive participants in the PL and MMPI groups on D1 and D84, directly after a 180-minute oral glucose tolerance test (OGTT; a relative insulin-stimulated state). We did not expect body composition changes in this short study but completed dual-energy x-ray absorptiometry (DXA) scans on D1 and D84 to confirm that any changes in muscle insulin sensitivity were not due to changes in body composition. While we did not expect change in global measures of glucose homeostasis in this short proof of concept study, based on the much longer time needed to see changes in these parameters in rodent studies, [9] we did assess hemoglobin A1c (A1c), calculated indices from the OGTT and used calculations from the homeostasis model assessment (HOMA) on D1 and D84 as comparators for future studies. Additional inflammatory markers measured on D1 and D84 included a cytokine array. Creatinine levels, the liver enzyme, AST, and a lipid panel as safety labs, and vital signs were measured on D1 and D84. Healthy control participants (controls), enrolled to provide comparison data for people without DM2, underwent screening and D1 visits only. 


\section{Muscle biopsies}

The skin was sterilely prepped and draped over the vastus lateralis $7 \mathrm{~cm}$ caudal to the medial patellar line. Lidocaine $(10 \mathrm{cc})$ was infused superficially then deep and the skin incised $5 \mathrm{~mm}$ with a scalpel. A trochanter was used to obtain $400 \mathrm{mg}$ of muscle that was immediately flash frozen in liquid nitrogen. Cyanoacrylates were used to close the wound followed by firm pressure and a compression wrap.

\section{MMP level and activity}

Plasma samples from the OGTT time zero were mixed for $30 \mathrm{~min}$ with $0.5 \mathrm{mg} / \mathrm{mL}$ of a charge-changing peptide substrate for MMP-2 and MMP-9 (Ac-NGDPVGLTAGAGK-NH2 [synthesized by Aapptec (Louisville, KY) and labeled with Bodipy FL-SE (Invitrogen)]) then loaded into $20 \%$ polyacrylamide gels and electrophoresed for 10 minutes prior to imaging as previously described. [21] A global assay for MMP activity levels was also performed on plasma samples using the SensoLyte ${ }^{\circledR} 520$ Generic MMP assay kit according to manufacturer's instructions (\#71158; AnaSpec, San Jose, CA.).

\section{Western immunoblotting}

Total proteins were extracted from muscle biopsies of 7 consecutive PL and 7 MMPI participants, in the presence of a mix of protease and phosphatase inhibitors (Thermo Scientific, Waltham, MA), which were separated by SDS-polyacrylamide gel electrophoresis (NuPAGE 4-12\% polyacrylamide Bis-Tris, Invitrogen) and transferred to PVDF membranes. Primary antibodies used for immunoblotting included total Akt and phospho Akt (Thr308); total PDK1 and phospho PDK1; total GSK3 $\beta$ and phospho GSK3 $\beta$; and GAPDH (Cell Signaling Technology, Danvers, MA.). Incubation of the immunoblots with horseradish peroxidase-conjugated secondary antibodies was carried out after membranes were incubated with their corresponding primary antibodies. The immunoblots were detected with Western lightning enhanced chemiluminescence detection reagent (PerkinElmer LAS, Inc., Norton, $\mathrm{OH}$ ). Band intensity was quantified with Scion ImageJ Software for personal computers (Scion, Frederick, MD) and expressed in arbitrary units. Day 84 values were normalized relative to baseline values equal to 1 . GAPDH was used as an internal standard.

\section{Oral glucose tolerance test (OGTT)}

The OGTT was performed once in all participants on D1 and on D84 in subjects with DM2 according to published guidelines. [22] Following an overnight fast, 75 grams of liquid carbohydrate was administered over a span of 5 minutes or less, with blood samples obtained via heparin lock at $-20,-10,0,15,30,60,90,120$, and
$180 \mathrm{~min}$ to determine glucose concentrations using a glucose analyzer (YSI Life Sciences, Yellow Spring, OH) and insulin levels. Plasma insulin levels were measured on stored frozen $\left(-80^{\circ} \mathrm{C}\right)$ samples by double-antibody sandwich electro chemiluminescence immunoassay (ECLIA; cat\#L45CA-1; Meso Scale Diagnostics [MSD], Gaithersburg, MD, USA) on Sector Imager 2400 (SI2400; MSD, Gaithersburg, MD, USA) by the UCSD Core laboratory. Data was analyzed for measures of insulin sensitivity and glucose utilization using published methods. [23]

\section{Glucose homeostasis}

Homeostasis Model Assessment of insulin secretion (HOMA-IS) was calculated as [ $20 \times$ (fasting insulin/7.175)/ (fasting glucose - 3.5)] [24,25] and HOMA-IR as [fasting insulin $(\mathrm{pmol} / \mathrm{l}) \times$ fasting glucose $(\mathrm{mmol} / \mathrm{l})] / 135$. The early secretory response of insulin to an oral glucose load ( $\Delta$ insulin30-0/ $\Delta$ glucose30-0) was calculated as $[\Delta$ insulin (30 $\mathrm{min}-0 \mathrm{~min})] /[\Delta$ glucose $(30 \mathrm{~min}-0 \mathrm{~min})]$ [26]. The incremental (above the baseline value) area under the curve $\left(A U C_{i}\right)$ for glucose and insulin was calculated using GRAPHPAD 5.0.

\section{Dual X-ray absorptiometry (DXA)}

Fat and fat-free mass were measured by DXA scan for whole-body composition on a Hologic Discovery W (Boston, MA) in the UCSD Clinical Trials and Research Institute (CTRI) and analyzed using software QDR DICOM for Windows XP.

\section{Other laboratory methods}

Serum AST, creatinine, total cholesterol, triglycerides, HDL, LDL, and A1c were measured at UCSD laboratories using established procedures.

\section{Biomarkers}

Assays were performed on plasma samples stored in -80 degree refrigerators according to manufacturer's kit instructions. MPO ELISA assay (\#30-6613A; ALPCO, Salem, NH), serum cytokines (Millipore, Billerica, MA), CRP ELISA (\#DCRP00; R\&D Systems, Inc., Minneapolis, MN).

\section{Statistical methods}

Randomization to PL or MMPI was in blocks of two using Research Randomizer. [27] Data are presented as average \pm SEM. Paired t-tests for pre- and posttreatment values, t-tests between the PL and MMPI groups, and AUC for insulin and glucose values obtained from the OGTT were performed using commercial software (GraphPad Prism version 5.00, GraphPad Software, San Diego California USA, www.graphpad.com). For MMP 2/9 activity analyses, a Wilcoxon signed rank test and sign tests were used for paired comparisons. For 3 
group comparisons (control vs. PL D1 vs. MMPI D1 or control vs. PL D84 vs. MMPI D84), a Kruskal-Wallis test was used, followed by post-hoc Mann-Whitney-Wilcoxon tests with a Bonferroni correction (SPSS). Post-hoc analyses were performed in order to determine which pair(s) of groups within that set explained the statistical significance. A p-value of .05 was used as the criterion for declaring statistical significance, with the exception that post-hoc tests used a p-value of 0.02 .

\section{Results}

\section{Study participants}

A total of 39 participants enrolled in this study including 15 controls without DM2 whose data served as comparison values for D1 data obtained from participants with DM2. Twenty-four controls were consented, three did not qualify and six failed to show for D1 procedures. Thirty-three participants with DM2 were enrolled, three did not qualify, three were unable to start the study due to medical problems and three failed to show for D1 procedures. Thirteen participants with DM2 were randomized to the PL group and eleven were randomized to the MMPI group. One subject in the PL group dropped out due to a skin yeast infection and one participant dropped from the MMPI group because of sun sensitivity. A second subject developed joint aches, night sweats, nausea and dizziness after MMPI treatment which resolved after stopping the drug. Two patients on MMPI developed nausea but completed the study.

There were no significant differences between baseline characteristics for the DM2 participants (Table 1). Statistically significant differences between the control and

Table 1 Baseline characteristics of study participants (Mean \pm SEM)

\begin{tabular}{lccc}
\hline Characteristics & Control & Placebo & Doxycycline \\
\hline Sample size & 15 & 13 & 11 \\
Age (years) & $48.7 \pm 3.1$ & $54.5 \pm 1.7$ & $55.3 \pm 1.9$ \\
Females (\%) & $5(0.33)$ & $2(0.15)$ & $2(0.18)$ \\
Weight (kg) & $79.1 \pm 3.6$ & $121.6 \pm 6.4^{* *}$ & $116.8 \pm 9.7^{* *}$ \\
Total Lean Mass (kg) & $52.5 \pm 17.5$ & $75.5 \pm 15.3^{* *}$ & $66.7 \pm 13.8^{*}$ \\
Total Fat Mass (kg) & $20.1 \pm 5.8$ & $40.7 \pm 10.3^{* *}$ & $37.8 \pm 13.3$ \\
BMl (kg/m²) & $26.8 \pm 0.86$ & $39.7 \pm 1.4^{* *}$ & $37.9 \pm 2.6^{* *}$ \\
Fasting Glucose (mg/dL) & $90.8 \pm 7.4$ & $178.2 \pm 43.8^{* *}$ & $149.4 \pm 53.4^{* *}$ \\
Insulin (ulU/mL) & $4.0 \pm 1.9$ & $41.0 \pm 35.1^{* *}$ & $39.4 \pm 43.2^{*}$ \\
A1c (\%) & $5.4 \pm 0.06$ & $8.5 \pm 0.4^{* *}$ & $8.2 \pm 0.5^{* *}$ \\
Race or ethnic group no. (\%) & & \\
Non-Hispanic white & $13(87)$ & $13(100)$ & $9(82)$ \\
Black & $1(7)$ & $0(0)$ & $1(9)$ \\
Hispanic & $1(7)$ & $0(0)$ & $1(9)$ \\
\hline
\end{tabular}

${ }^{*} \mathrm{P}<0.05$ versus control , ${ }^{* *} \mathrm{P}<0.001$ versus control.
DM2 groups were found for weight, BMI, fasting glucose and insulin, and A1c (Table 1).

\section{Markers of inflammation}

Baseline levels of CRP were higher in the PL and MMPI groups $(\mathrm{P}<0.05$; Table 2$)$ compared to controls. There was no significant change in $\mathrm{CRP}$ in the $\mathrm{PL}$ group $(\mathrm{P}=0.8)$ but a significant decrease in CRP in the MMPI group from D1 to D84 ( $<<0.05$; Table 2; Figure 1$)$. Baseline levels of MPO were significantly higher in the PL and MMPI groups on D1 and in the PL group on D84 compared to controls (Figure 1). MPO in the PL group did not change significantly between D1 and D84 $(\mathrm{P}=0.4)$. MPO significantly decreased in the MMPI group from D1 to D84 ( $\mathrm{P}=0.001$; Table 2; Figure 1). There was no significant change from D1 to D84 for cytokines in the PL or MMPI groups except for a significant decrease in IL-10 in the PL group $(\mathrm{P}<0.04$; Table 2$)$.

\section{Insulin signaling}

There was no difference between the participants in the PL $(n=7)$ or MMPI group $(n=7)$ for age $(54.0 \pm 7.1 y ; 56 . \pm 5.3 y)$, weight $(120.8 \pm 22.7 \mathrm{~kg} ; 113.7 \pm 35.2 \mathrm{~kg})$, BMI $\left(40.0 \pm 6.2 \mathrm{~kg} / \mathrm{m}^{2}\right.$; $\left.35.7 \pm 7 \mathrm{~kg} / \mathrm{m}^{2}\right)$ or A1c $(9.0 \pm 1.5 \% ; 7.7 \pm 1.7 \%)$ for which muscle was assessed for activation of insulin signaling mediators. In the PL group, there were no statistically significant differences in the phosphorylation status of PDK1, Akt, or GSK3 $\beta$ from D1 to D84 (Figure 2). In contrast, in the MMPI group there was a statistically significant increase in the phosphorylation status of PDK1, Akt, and GSK3 $\beta$ on D84 compared to D1 (Figure 2).

\section{Vital signs and lipids}

There was no significant change in systolic or diastolic blood pressure from D1 to D84 in the PL or MMPI group (Table 2). There was no significant change in any parameter of the lipid profile for the MMPI group (Table 2). In the PL group, total cholesterol decreased significantly $(P=0.015)$ as did HDL $(P=0.009)$ from D1 to D84. There was no significant change in triglycerides $(P=0.3)$ or $L D L$ $(\mathrm{P}=0.08)$ in the PL group from D1 to D84.

\section{AST and creatinine}

There was no significant change in creatinine level in the PL $(\mathrm{P}=1.0)$ or MMPI group $(\mathrm{P}=0.7$; Table 2$)$ or AST level in the PL $(P=0.6)$ or MMPI group $(P=0.1$; Table 2) from D1 to D84.

\section{Body composition}

There was no significant change in weight in the PL $(121.6 \pm 6.4$ vs. $122.6 \pm 6.4 \mathrm{~kg})$ or MMPI group $(125.4 \pm 12.2$ vs. $126.7 \pm 12.7 \mathrm{~kg} ; \mathrm{P}=0.2$ for both), lean mass in the PL $(75.5 \pm 15.3$ vs. $74.5 \pm 14.1 \mathrm{~kg})$ or MMPI 
Table 2 Outcome measures on D1 and D84 (Mean \pm SEM)

\begin{tabular}{|c|c|c|c|c|c|}
\hline \multirow[t]{2}{*}{ Measures } & \multirow[t]{2}{*}{ Control } & \multicolumn{2}{|c|}{ Placebo Group $(n=11)$} & \multicolumn{2}{|c|}{ MMPI Group $(n=9)$} \\
\hline & & D1 & D84 & D1 & D84 \\
\hline CRP & $1.9 \pm 0.8$ & $5.9 \pm 1.8^{*}$ & $6.2 \pm 1.8^{*}$ & $7.4 \pm 3.3^{*}$ & $2.3 \pm 0.4 \dagger$ \\
\hline MPO & $68.8 \pm 6.6$ & $88.6 \pm 6.0^{*}$ & $94.5 \pm 11.3^{*}$ & $110.2 \pm 15.3^{*}$ & $91.6 \pm 13.6+$ \\
\hline $\mathrm{SBP}(\mathrm{mmHg})$ & $115.7 \pm 19.6$ & $127.7 \pm 5.7$ & $133.4 \pm 4.8$ & $136.2 \pm 6.5$ & $137.5 \pm 5.8$ \\
\hline $\mathrm{DBP}(\mathrm{mmHg})$ & $72.0 \pm 10.5$ & $77.0 \pm 10.1$ & $77.8 \pm 11$ & $77.8 \pm 8.0$ & $77.4 \pm 11.2$ \\
\hline $\mathrm{TC}(\mathrm{mg} / \mathrm{dL})$ & $177.5 \pm 28.0$ & $178.8 \pm 13$ & $152.2 \pm 6$ & $177.9 \pm 14$ & $165.1 \pm 10$ \\
\hline $\mathrm{HDL}(\mathrm{mg} / \mathrm{dL})$ & $54.0 \pm 15.2$ & $40.5 \pm 3.2^{* *}$ & $36.58 \pm 3.0^{* *}$ & $38.44 \pm 2.6^{* *}$ & $38.44 \pm 3.2^{* *}$ \\
\hline $\mathrm{LDL}(\mathrm{mg} / \mathrm{dL})$ & $106.7 \pm 22.2$ & $99.6 \pm 12$ & $82 \pm 7$ & $86 \pm 9$ & $86.4 \pm 8.8$ \\
\hline $\mathrm{TG}(\mathrm{mg} / \mathrm{dL})$ & $84.4 \pm 39.0$ & $209.1 \pm 30^{* *}$ & $191.5 \pm 36^{* *}$ & $235.9 \pm 48^{* *}$ & $188.6 \pm 37^{* *}$ \\
\hline AST (IU/L) & $22.8 \pm 7.7$ & $30.8 \pm 4.1$ & $33.0 \pm 6.1$ & $24.4 \pm 2.9$ & $20.1 \pm 1.4$ \\
\hline Creatinine $(\mathrm{mg} / \mathrm{dL})$ & $0.9 \pm 0.2$ & $0.83 \pm 0.05$ & $0.83 \pm 0.07$ & $0.91 \pm 0.05$ & $0.92 \pm 0.06$ \\
\hline Fasting glucose (mmol/L) & $5.1 \pm 1.2$ & $9.4 \pm 0.5^{* *}$ & $8.8 \pm 0.6^{* *}$ & $8.0 \pm 0.7^{* *}$ & $8.0 \pm 1.0^{* *}$ \\
\hline $\mathrm{A} 1 \mathrm{C}(\%)$ & $5.4 \pm 0.2$ & $8.6 \pm 0.4$ & $8.0 \pm 0.3$ & $8.3 \pm 0.6$ & $7.8 \pm 0.4$ \\
\hline$A \cup C_{i}$ & $0.25 \pm 0.07$ & $0.57 \pm 0.16$ & $0.57 \pm 0.13^{*}$ & $0.89 \pm 0.31^{*}$ & $0.71 \pm 0.29$ \\
\hline HOMA-IS & $53.4 \pm 7.6$ & $135.3 \pm 32.7$ & $164 \pm 46.8$ & $244.9 \pm 98.5$ & $433.1 \pm 272.1$ \\
\hline HOMA-IR & $1.06 \pm 0.1$ & $21 \pm 5.1$ & $26 \pm 9.8$ & $14.3 \pm 4.7$ & $23.0 \pm 11.1$ \\
\hline$\Delta \mid 30-0 / \Delta \mathrm{G} 30-0$ & $98 \pm 17.5$ & $37.3 \pm 51.1$ & $54.3 \pm 30$ & $73.7 \pm 35.9$ & $16.6 \pm 20.1+$ \\
\hline Global MMP (uM 5-FAM/h-ug protein) & $47.2 \pm 3.2$ & $43.8 \pm 4$ & $56.6 \pm 10.1$ & $56.8 \pm 13.3$ & $59.7 \pm 4.3$ \\
\hline $\mathrm{IL}-1 \beta(\mathrm{pg} / \mathrm{ml})$ & $0.15 \pm 0.06$ & $0.14 \pm 0.05$ & $0.13 \pm 0.04$ & $0.27 \pm 0.15$ & $0.46 \pm 0.22$ \\
\hline IL-2 (pg/ml) & $1.8 \pm 0.6$ & $1.1 \pm 0.4$ & $0.9 \pm 0.3$ & $0.7 \pm 0.3$ & $1.1 \pm 0.4$ \\
\hline IL-4 (pg/ml) & $10.0 \pm 2.5$ & $7.3 \pm 2.1$ & $9.9 \pm 4.5$ & $9.8 \pm 4.2$ & $7.4 \pm 2.3$ \\
\hline IL-6 (pg/ml) & $6.9 \pm 1.5$ & $5.3 \pm 1.9$ & $8.4 \pm 3.5$ & $10.9 \pm 4.3$ & $12.9 \pm 5.4$ \\
\hline IL-8 (pg/ml) & $2.4 \pm 0.4$ & $2.8 \pm 0.5$ & $3.2 \pm 0.5$ & $4.8 \pm 0.9$ & $5.8 \pm 1.0$ \\
\hline $\mathrm{IL}-10(\mathrm{pg} / \mathrm{ml})$ & $16.6 \pm 3.8$ & $15.1 \pm 3.5$ & $11.3 \pm 3.0+$ & $16.4 \pm 5.2$ & $16.8 \pm 3.8$ \\
\hline TNF-a (pg/ml) & $4.7 \pm 0.6$ & $4.4 \pm 0.4$ & $4.4 \pm 0.7$ & $6.6 \pm 1.3$ & $5.8 \pm 1.1$ \\
\hline
\end{tabular}

${ }^{*} \mathrm{P}<0.05$ versus control, ${ }^{* *} \mathrm{P}<0.01$ versus control, $+\mathrm{P}<0.05$ vs. D1.

group $(66.7 \pm 13.8$ vs. $65.9 \pm 14.2 \mathrm{~kg} ; \mathrm{P}=0.8$ for both $)$ or fat mass in the PL $(40.7 \pm 10.3$ vs. $41.5 \pm 10.1)$ or MMPI group $(37.8 \pm 13.3$ vs. $39.8 \pm 15.1 \mathrm{~kg} ; \mathrm{P}=0.3$ for both) between $\mathrm{D} 1$ and D84.

\section{Glucose homeostasis}

The fasting glucose level was significantly lower in the control group compared to the MMPI and PL groups on D1 and D84 (Table 2). There was no significant change in A1c between D1 and D84 for the PL or MMPI group (Table 2). The mean percent change in A1c values between the MMPI and PL groups was not significantly different (data on file; $\mathrm{P}=0.6$ ). The $\mathrm{AUC}_{\mathrm{i}}$ did not change from D1 to D84 for the PL or MMPI groups (Table 2). The $\mathrm{AUC}_{\mathrm{i}}$ for the PL group was not significantly different compared to the control group on D1 but became significantly higher at D84 (Table 2). The $\mathrm{AUC}_{\mathrm{i}}$ for the MMPI group was significantly higher than the control group at D1 but became non-significantly different at D84 (Table 2). There was no change in the $\Delta \mathrm{I} 30-0 /$
$\Delta \mathrm{G} 30-0$ in the PL group from $\mathrm{D} 1$ to $\mathrm{D} 84$. The $\Delta \mathrm{I} 30-0 /$ $\triangle$ G30-0 significantly decreased in the MMPI group from D1 to D84 (Table 2). There was no significant difference for HOMA-IS for the PL group at D1 compared to D84 ( $\mathrm{P}=0.4$; Table 2). The HOMA-IS trended higher for the MMPI group on D84 compared to D1 ( $\mathrm{P}=0.05$; Table 2). There was no significant change in the HOMA-IR for the PL $(\mathrm{P}=0.4)$ or MMPI groups $(\mathrm{P}=0.07)$ from D1 to D84 (Table 2).

\section{MMP level and activity}

There was no difference in global MMP level between the control group and the PL or MMPI groups on D1 or D84, and no change in the global MMP level in the PL or MMPI groups from D1 to D84 (Table 2).

MMP 2/9 activity was significantly lower in the control group compared to the PL and MMPI groups on D1, and PL group on D84 (Figure 3). There was no difference in MMP 2/9 activity between the PL and MMPI groups on D1 or D84 (Figure 3). 

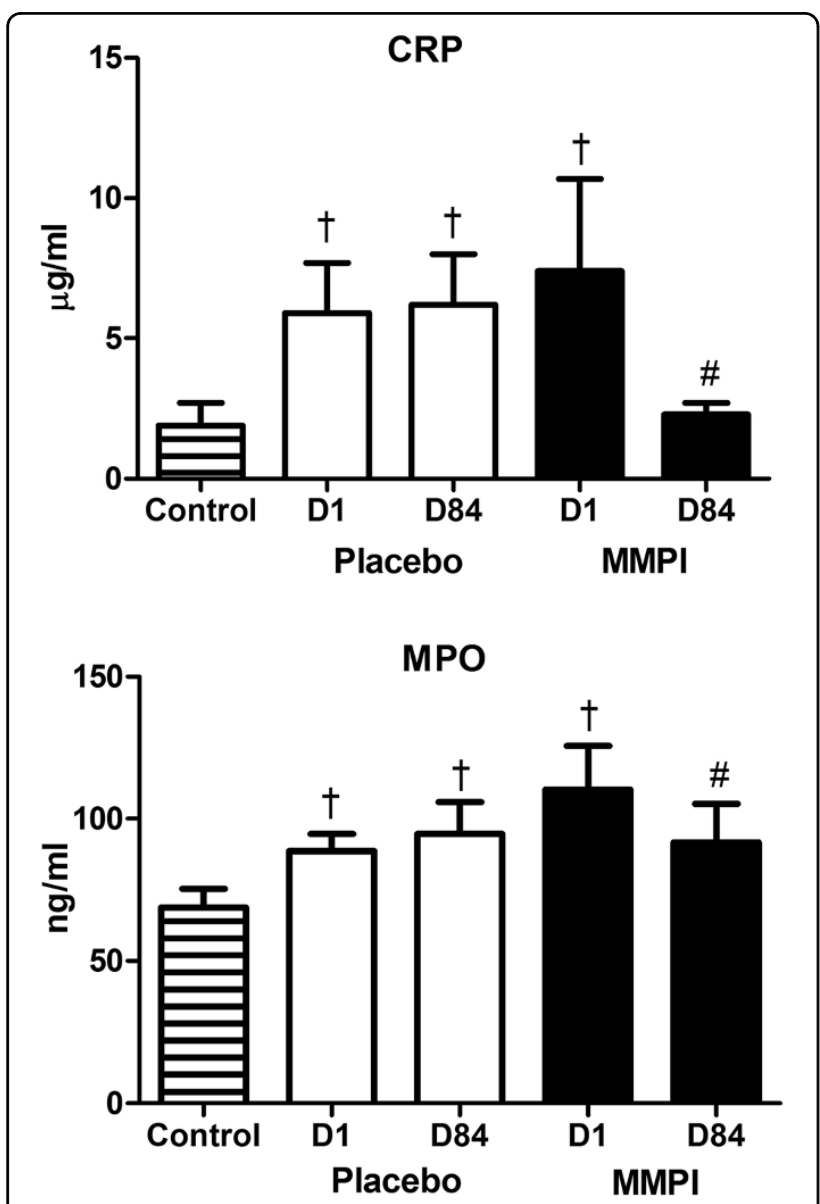

Figure $1 \mathrm{MPO}$ and CRP levels for the Control, PL, and MMPI groups. MPO and CRP levels for the Control group at D1, and the $\mathrm{PL}$ and MMPI groups at D1 and D84. $+\mathrm{P}<0.05$ versus Control group. \# $<0.05$ versus D1 of the MMPI group.

\section{Discussion}

This proof of concept study confirmed that in only 12 weeks, the MMPI, doxycycline, improved global measures of inflammation and muscle insulin sensitivity in people with DM2.

Global markers of inflammation, CRP and MPO levels in plasma, were significantly higher in obese participants with diabetes before treatment compared to controls, supporting the pro-inflammatory state expected in this population. CRP is a global measure of inflammation and oxidative stress even in apparently "healthy" populations [28] and MPO is a lysosomal enzyme in neutrophils that has been associated with coronary plaque instability [29] and therefore increased cardiovascular risk. [30] As expected, MPO levels have been shown to be higher in people with diabetes compared to controls. [31] MMPI treatment resulted in a significant decrease in both CRP and MPO by the end of the study whereas PL had no effect, supporting the anti-inflammatory properties of doxycycline. While medications such as
HMG coA reductase inhibitors ("statins") or aspirin are known to affect inflammatory markers (i.e. CRP, MPO), participants did not change medications during the study and so drug effects for our study participants should have been stable. Other than a significant decrease in the anti-inflammatory cytokine IL-10 in the PL group, there were no significant changes in cytokine levels in the PL or MMPI group. Changes in expression of cytokines after MMPI may be better measured in tissues rather than blood.

To test for an effect of MMPI on insulin sensitivity, three different kinases in the insulin signaling cascade in muscle (PDK1, Akt, GSK3 $\beta$ ) were examined in muscle biopsies obtained directly after an OGTT (a relative insulin-stimulated state). In the MMPI treated group, there was a significant increase in the phosphorylation status of all of three kinases, indicating an improvement in the insulin sensitivity of the muscle, with no change in the PL group. Data are needed to link the global decrease in inflammation with improved insulin sensitivity in muscle.

Golub et al. first demonstrated inhibition of MMPs by tetracyclines in people with diabetes. $[14,17,32]$ The decision to use doxycycline in this study was based on these data, its broad spectrum MMPI [9,12,13], and its successful MMPI in a number of pathological states [16] including an animal model of diabetes. [9] The literature provides additional evidence that doxycycline can inhibit the activity of other classes of proteases such as plasmin (a serine protease) [33], and act as an oxygen radical scavenger. [34] Doxycycline is also known to be relatively well tolerated with minor side-effects. This study resulted in one case of sun sensitivity with doxycycline use, two cases of nausea and one patient developed serum sickness. Serum sickness like syndrome is known to occur with the use of the tetracyclines, minocycline [35] and doxycycline. [36]

Dosing schemes of MMPI vary widely from $40 \mathrm{mg}$ daily [37] and upwards, including the usual adult antibiotic dose of $100 \mathrm{mg}$ twice daily used in this proof of concept study, a dose reported to reduce CRP levels during inflammation associated with infection [38]. That doxycycline improved insulin sensitivity in muscle but not global glucose homeostasis in people with DM2 suggests the need for larger and longer clinical trials, as well as a dose finding study as lower amounts of doxycycline $(40 \mathrm{mg}$ daily) have been shown to significantly reduce CRP in people with cardiovascular disease over 6 months [39].

The proposed mechanism by which doxycycline improves insulin sensitivity, is by inhibition of inflammatory MMPs that cleave the extracellular insulin receptor domain, thereby inactivating the receptor. [9] Doxycycline and other MMPI have been shown to inhibit cleavage of other receptors including vascular endothelial 


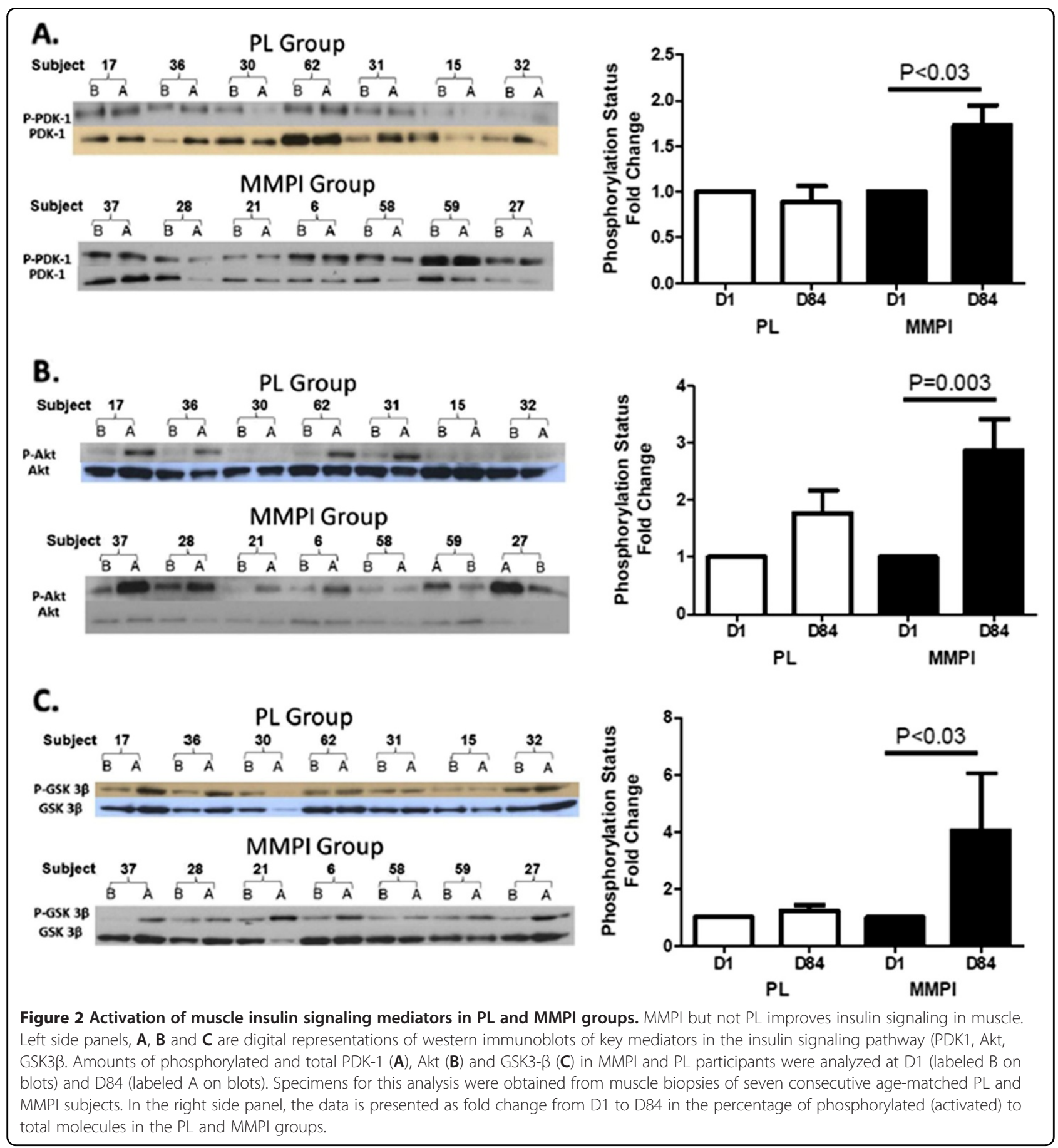

growth factor receptor 2 (VEGFR-2). [40] We did not find a difference in global MMP levels between control participants and those with DM2, and no change in global MMP levels after MMPI; this may be due to the relatively low sensitivity of this technique. Using a new and more sensitive technology to measure MMP 2/9 activity, [21] we found higher MMP 2/9 activity levels in all obese study participants with diabetes compared to the control group prior to treatment, in agreement with others. [2,19,41] This difference is likely due to the higher sensitivity of the electrophoretic zymographic technique and the fact that it does not require separation of blood cells from plasma. [21] However, we found no significant effect of doxycycline on blood levels of MMP 2/9 activity at study completion using this new technique. We hypothesize that although we found improvements in insulin sensitivity, the time of the study was not long enough to see a reduction in these MMP 


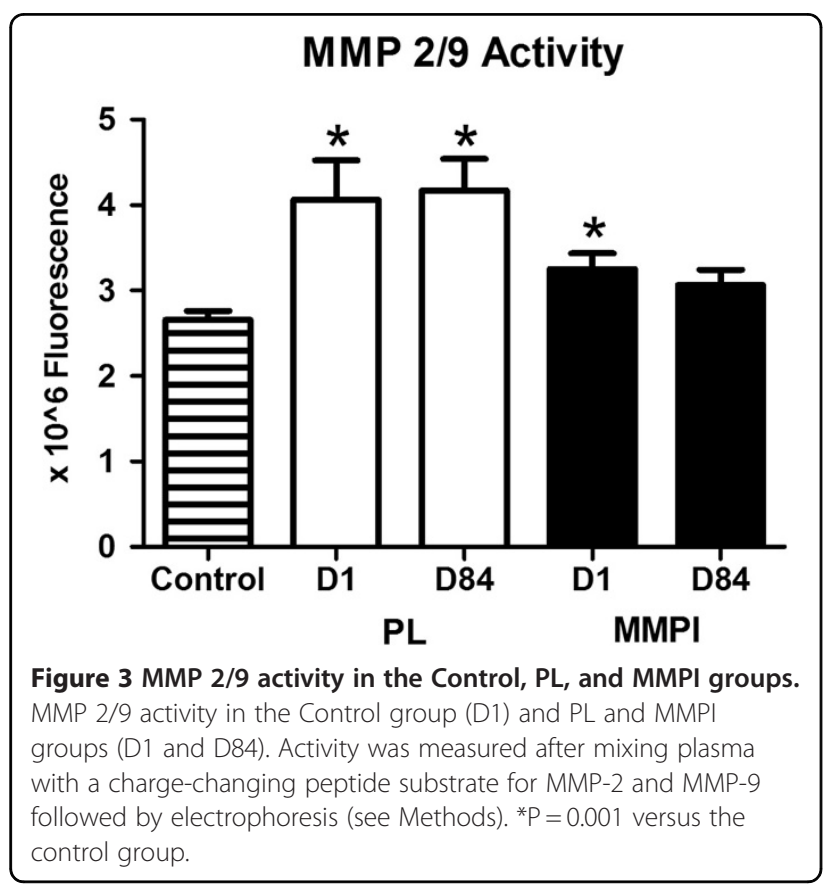

levels in people with severe insulin resistance, and that gene expression of MMPs and TIMP levels need to be measured along with MMPs. During our study, it was reported that blood MMP 2/9 activity levels do not change in participants with DM2 during an OGTT. [42] It appears that MMP blood activity, at present, could be more useful in screening for whether a patient should take MMPI (after observing elevated MMP activity), but isn't as sensitive for monitoring the effect of treatment after a glucose load. It may require a stronger stimulus than glucose alone, such as a mixed meal, to induce measureable changes in MMP levels in the blood.

There are many factors that can affect MMP activity in blood, in addition to DM2. The development of more specific protease substrates that better differentiate the activity from individual types of MMPs and other proteases would be useful in studies such as these. From animal and in vitro studies, it may be that the best way to measure the effect of MMPI on MMP activity is not through blood samples but in tissues such as adipose tissue, where MMP activity is increased in obesity and tissue inhibitors of MMPs (TIMPs) are decreased. [2] Studies are ongoing to test whether MMPI can affect MMP and TIMP levels, and insulin receptor integrity in humans.

Commonly measured clinical parameters of DM control were evaluated in this study including weight, A1c, triglycerides, and blood pressure which did not change in either the MMPI or PL groups. The early secretory response of insulin to an oral glucose load, however, was significantly lower after MMPI treatment as was the secretion of insulin measured by the HOMA-IS, and the
$\mathrm{AUC}_{\mathrm{i}}$ was improving in the MMPI group but not the PL group. While there was a significant increase in activation of molecules in the insulin signaling pathways during this period, the time it takes to see a clinically significant effect on overall glucose control is likely longer. Improvements in global measures of glucose homeostasis by MMPI required 6 months in rats [9] and may be even longer in a more genetically mixed population of people. DM2 participants in this trial were on antihypertensive, lipid lowering and hypoglycemic medications which may have affected changes in insulin sensitivity. Larger numbers of participants with both new onset DM or impaired glucose tolerance or impaired fasting glucose with minimal medication use and a longer trial will be important in determining if there are any benefits to MMPI use in the treatment of inflammation and reduced insulin sensitivity in humans. If doxycycline were to be used long-term, studies on development of resistance or changes in gastrointestinal flora would be helpful to understand the contribution of these changes to markers of inflammation and insulin sensitivity. Additional limitations of this study include the lack of assessment of tissue MMPs, and small study size.

\section{Conclusions}

Treatment of obese diabetics with doxycycline for 12 weeks significantly decreased serum markers of inflammation while increasing muscle tissue insulin sensitivity; these data suggest that longer studies in people should be explored. Studies with larger numbers of patients, longer treatment periods of escalating doses of doxycycline and a detailed evaluation of the underlying mechanisms leading to improvements in inflammation and insulin sensitivity may provide greater insight into the potential for using doxycycline and other MMP inhibitors in human disease.

\section{Abbreviations}

MMP: Matrix metalloproteinase; PL: Placebo; MMPI: MMP inhibitor; DM2: Type 2 diabetes; PDK1: 3'phosphoinositide kinase-1; PKB/Akt: Protein kinase B; GSK3 B: Glycogen synthase kinase 3B; PI3K: Phosphatidyl inositol-3-kinase; GLUT4: Glucose transporter; CRP: C-reactive protein; MPO: Myeloperoxidase; OGTT: Oral glucose tolerance test; DXA: Dual-energy x-ray absorptiometry; A1c: Hemoglobin A1c; HOMA: Homeostasis model assessment.

\section{Competing interests}

GWSS holds interest in InhibeX, a company that has licensed MMP treatment from UCSD.

\section{Authors' contributions}

KH, GWSS, FV conceptualized this study and developed the initial study structure. KH, KF, CT, and MB conducted subject visits, collected samples, and input data for analyses, statistics and writing the paper. RL, MT, and MH conducted MMP 2/9 activity data, conducted statistics and wrote methods for these data. MTG conducted muscle and MPO assays and wrote methods and results for these data. KF, KH, CT were involved in writing the paper including statistics and figure development. All authors read and approved the final manuscript. 


\section{Acknowledgements}

This study received Core and research support from the UCSD NIDDK Diabetes and Endocrinology Research Center Grant, the UCSD General Clinical Research Center by Public Health Grant, Ruth L. Kirschstein National Research Service Award, NIH Grant R01, NIH Fellowship support, and storage, facility and computer services at the VA San Diego Healthcare System.

\section{Author details}

'Veteran's Affairs, San Diego Healthcare System, 3350 La Jolla Village Drive (111 G), San Diego, CA 92161, USA. ${ }^{2}$ Department of Medicine, Division of Endocrinology \& Metabolism, University of California, 9500 Gilman Drive, \#0673 La Jolla, San Diego, CA 92093, USA. 르epartment of Bioengineering, University of California, 9500 Gilman Drive, \#0412 La Jolla, San Diego, CA 92093, USA. ${ }^{4}$ Department of Pharmacology, University of California, 9500 Gilman Drive, \#0636 La Jolla, San Diego, CA 92093, USA. ${ }^{5}$ Veteran's Medical Research Foundation, 3350 La Jolla Village Drive (111 G), San Diego, CA 92161, USA.

Received: 17 June 2012 Accepted: 10 September 2012 Published: 1 October 2012

\section{References}

1. Curat CA, Wegner V, Sengenes C, Miranville A, Tonus C, Busse R, Bouloumie $A$ : Macrophages in human visceral adipose tissue: increased accumulation in obesity and a source of resistin and visfatin. Diabetologia 2006, 49:744-7. Epub 2006 Feb 23.

2. Chavey C, Mari B, Monthouel MN, Bonnafous S, Anglard P, Van Obberghen E, Tartare-Deckert S: Matrix metalloproteinases are differentially expressed in adipose tissue during obesity and modulate adipocyte differentiation. J Biol Chem 2003, 278:11888-96. Epub 2003 Jan 15.

3. Derosa G, Ferrari I, D'Angelo A, Tinelli C, Salvadeo SA, Ciccarelli L, Piccinni MN, Gravina A, Ramondetti F, Maffioli P, Cicero AF: Matrix metalloproteinase-2 and -9 levels in obese patients. Endothelium 2008, 15:219-24.

4. Catalan V, Gomez-Ambrosi J, Rodriguez A, Ramirez B, Silva C, Rotellar F, Gil MJ, Cienfuegos JA, Salvador J, Fruhbeck G: Increased adipose tissue expression of lipocalin-2 in obesity is related to inflammation and matrix metalloproteinase-2 and metalloproteinase-9 activities in humans. J Mol Med 2009, 87:803-13. Epub 2009 May 23.

5. Andarawewa KL, Rio M-C: New Insights into MMP function in Adipogenesis. In The Cancer Degradome. Edited by Edwards D, Høyer-Hansen G, Blasi F, Slo BF. New York: Springer; 2008:361-72.

6. Mieczkowska J, Mosiewicz J, Barud W, Kwasniewski W: Changes in the activity of connective tissue matrix enzymes in the metabolic syndrome. Arch Med Sci 2011, 7:634-41.

7. Ress C, Tschoner A, Ciardi C, Laimer MW, Engl JW, Sturm W, Weiss H, Tilg H, Ebenbichler CF, Patsch JR, Kaser S: Influence of significant weight loss on serum matrix metalloproteinase (MMP)-7 levels. Eur Cytokine Netw 2010, 21:65-70.

8. Rodrigues SF, Tran ED, Fortes ZB, Schmid-Schonbein GW: Matrix metalloproteinases cleave the beta2-adrenergic receptor in spontaneously hypertensive rats. Am J Physiol Heart Circ Physiol 2010, 299:H25-35. Epub 2010 Apr 9.

9. DeLano FA, Schmid-Schonbein GW: Proteinase activity and receptor cleavage: mechanism for insulin resistance in the spontaneously hypertensive rat. Hypertension 2008, 52:415-23.

10. Lucotti P, Monti LD, Setola E, Galluccio E, Gatti R, Bosi E, Piatti P: Aerobic and resistance training effects compared to aerobic training alone in obese type 2 diabetic patients on diet treatment. Diabetes Res Clin Pract 2011, 94:395-403. Epub 2011 Sep 3.

11. Madsen EL, Bruun JM, Skogstrand K, Hougaard DM, Christiansen T, Richelsen B: Long-term weight loss decreases the nontraditional cardiovascular risk factors interleukin-18 and matrix metalloproteinase-9 in obese subjects. Metabolism 2009, 58:946-53.

12. Zhang P, Yang YJ, Chen X, Ruan YM, Zhou YW, Tian Y, Chen ZJ: Comparison of doxycycline, losartan, and their combination on the expression of matrix metalloproteinase, tissue inhibitor of matrix metalloproteinase, and collagen remodeling in the noninfarcted myocardium after acute myocardial infarction in rats. Zhongguo Yi Xue Ke Xue Yuan Xue Bao 2005, 27:53-61.
13. Li DQ, Shang TY, Kim HS, Solomon A, Lokeshwar BL, Pflugfelder SC: Regulated expression of collagenases MMP-1, -8 , and -13 and stromelysins MMP-3, -10 , and -11 by human corneal epithelial cells. Invest Ophthalmol Vis Sci 2003, 44:2928-36.

14. Ryan ME, Usman A, Ramamurthy NS, Golub LM, Greenwald RA: Excessive matrix metalloproteinase activity in diabetes: inhibition by tetracycline analogues with zinc reactivity. Curr Med Chem 2001, 8:305-16.

15. Chung AW, Yang HH, Radomski MW, van Breemen C: Long-term doxycycline is more effective than atenolol to prevent thoracic aortic aneurysm in marfan syndrome through the inhibition of matrix metalloproteinase-2 and -9. Circ Res 2008, 102:e73-85.

16. Hackmann AE, Rubin BG, Sanchez LA, Geraghty PA, Thompson RW, Curci JA: A randomized, placebo-controlled trial of doxycycline after endoluminal aneurysm repair. J Vasc Surg 2008, 48:519-26. discussion 26.

17. Sorsa T, Ingman T, Suomalainen $K$, Halinen S, Saari H, Konttinen YT, Uitto VJ, Golub LM: Cellular source and tetracycline-inhibition of gingival crevicular fluid collagenase of patients with labile diabetes mellitus. J Clin Periodontol 1992, 19:146-9.

18. Dipl-Pharm SG, Zierath JR: Tackling the Insulin-signalling Cascade. Canadian Journal of Diabetes 2005, 29:239-45.

19. Unal R, Yao-Borengasser A, Varma V, Rasouli N, Labbate C, Kern PA, Ranganathan G: Matrix metalloproteinase-9 is increased in obese subjects and decreases in response to pioglitazone. J Clin Endocrinol Metabol 2010, 95:2993-3001. Epub 2010 Apr 14.

20. Friese RS, Rao F, Khandrika S, Thomas B, Ziegler MG, Schmid-Schonbein GW, O'Connor DT: Matrix metalloproteinases: discrete elevations in essential hypertension and hypertensive end-stage renal disease. Clin Exp Hypertens 2009, 31:521-33.

21. Lefkowitz RB, Schmid-Schonbein GW, Heller MJ: Whole blood assay for elastase, chymotrypsin, matrix metalloproteinase-2, and matrix metalloproteinase-9 activity. Anal Chem 2010, 82:8251-8.

22. French $L R$, Boen JR, Martinez AM, Bushhouse SA, Sprafka JM, Goetz FC: Population-based study of impaired glucose tolerance and type II diabetes in Wadena. Minnesota Diabetes 1990, 39:1131-7.

23. Abdul-Ghani MA, Matsuda M, Balas B, DeFronzo RA: Muscle and Liver Insulin Resistance Indexes Derived From the Oral Glucose Tolerance Test. Diabetes Care 2007, 30:89-94.

24. Antuna-Puente B, Faraj M, Karelis AD, Garrel D, Prud'homme D, Rabasa-Lhoret R, Bastard JP: HOMA or QUICKI: is it useful to test the reproducibility of formulas? Diabetes Metab 2008, 34:294-6. Epub 2008 May 12.

25. Nagaretani H, Nakamura T, Funahashi T, Kotani K, Miyanaga M, Tokunaga K, Takahashi M, Nishizawa H, Kishida K, Kuriyama H, Hotta K, Yamashita S, Matsuzawa Y: Visceral fat is a major contributor for multiple risk factor clustering in Japanese men with impaired glucose tolerance. Diabetes Care 2001, 24:2127-33.

26. Phillips DI, Clark PM, Hales CN, Osmond C: Understanding oral glucose tolerance: comparison of glucose or insulin measurements during the oral glucose tolerance test with specific measurements of insulin resistance and insulin secretion. Diabet Med 1994, 11:286-92.

27. Urbaniak GC, Plous S: Research Randomizer (Version 3.0) [Computer software]; 2011. Retrieved on April 22, 2010, from http://www.randomizer.org.: Social Psychology Network.

28. Kelishadi R, Sharifi M, Khosravi A, Adeli K: Relationship between C-reactive protein and atherosclerotic risk factors and oxidative stress markers among young persons 10-18 years old. Clin Chem 2007, 53:456-64. Epub 2007 Jan 26

29. Roman RM, Camargo PV, Borges FK, Rossini AP, Polanczyk CA: Prognostic value of myeloperoxidase in coronary artery disease: comparison of unstable and stable angina patients. Coron Artery Dis 2010, 21:129-36.

30. Karakas M, Koenig W, Zierer A, Herder C, Rottbauer W, Baumert J, Meisinger C, Thorand B: Myeloperoxidase is Associated with Incident Coronary Heart Disease Independently of Traditional Risk Factors: Results from the MONICA/KORA Augsburg Study. J Intern Med, 2011:1365-2796.

31. Moldoveanu E, Tanaseanu C, Tanaseanu S, Kosaka T, Manea G, Marta DS, Popescu LM: Plasma markers of endothelial dysfunction in type 2 diabetics. Eur J Intern Med 2006, 17:38-42.

32. Golub LM, Lee HM, Lehrer G, Nemiroff A, McNamara TF, Kaplan R, Ramamurthy NS: Minocycline reduces gingival collagenolytic activity 
during diabetes. Preliminary observations and a proposed new mechanism of action. J Periodontal Res 1983, 18:516-26.

33. Griffin MO, Jinno M, Miles LA, Villarreal FJ: Reduction of myocardial infarct size by doxycycline: a role for plasmin inhibition. Mol Cell Biochem 2005, 270:1-11.

34. Kraus RL, Pasieczny R, Lariosa-Willingham K, Turner MS, Jiang A, Trauger JW: Antioxidant properties of minocycline: neuroprotection in an oxidative stress assay and direct radical-scavenging activity. J Neurochem 2005, 94:819-27.

35. Puyana J, Urena V, Quirce S, Fernandez-Rivas M, Cuevas M, Fraj J: Serum sickness-like syndrome associated with minocycline therapy. Allergy 1990, 45:313-5.

36. Shapiro LE, Knowles SR, Shear NH: Comparative safety of tetracycline, minocycline, and doxycycline. Arch Dermatol 1997, 133:1224-30.

37. Golub LM, Lee HM, Stoner JA, Sorsa T, Reinhardt RA, Wolff MS, Ryan ME, Nummikoski PV, Payne JB: Subantimicrobial-dose doxycycline modulates gingival crevicular fluid biomarkers of periodontitis in postmenopausal osteopenic women. J Periodontol 2008, 79:1409-18.

38. Daniels JM, Schoorl M, Snijders D, Knol DL, Lutter R, Jansen HM, Boersma WG: Procalcitonin vs C-reactive protein as predictive markers of response to antibiotic therapy in acute exacerbations of COPD. Chest 2010, 138:1108-15.

39. Brown DL, Desai KK, Vakili BA, Nouneh C, Lee HM, Golub LM: Clinical and biochemical results of the metalloproteinase inhibition with subantimicrobial doses of doxycycline to prevent acute coronary syndromes (MIDAS) pilot trial. Arterioscler Thromb Vasc Biol 2004 24:733-8. Epub 2004 Feb 12.

40. Tran ED, Yang M, Chen A, Delano FA, Murfee WL, Schmid-Schonbein GW: Matrix Metalloproteinase Activity Causes VEGFR-2 Cleavage and Microvascular Rarefaction in Rat Mesentery. Microcirculation, 2011:1549-8719.

41. Dominguez-Rodriguez A, Abreu-Gonzalez P, Garcia-Gonzalez MJ, Kaski JC High serum matrix metalloproteinase-9 level predict increased risk of in-hospital cardiac events in patients with type 2 diabetes and ST segment elevation myocardial infarction. Atherosclerosis 2008, 196:365-71. Epub 2006 Dec 11

42. Lewandowski $K C$, Banach $E$, Lewinski A: Concentrations of matrix metalloproteinase 2 and 9 in patients with type 2 diabetes and in non-diabetic controls. Endocr Abstr 2009, 19:P121.

doi:10.1186/1476-9255-9-35

Cite this article as: Frankwich et al:: Proof of Concept: Matrix metalloproteinase inhibitor decreases inflammation and improves muscle insulin sensitivity in people with type 2 diabetes. Journal of Inflammation 2012 9:35.

\section{Submit your next manuscript to BioMed Central and take full advantage of:}

- Convenient online submission

- Thorough peer review

- No space constraints or color figure charges

- Immediate publication on acceptance

- Inclusion in PubMed, CAS, Scopus and Google Scholar

- Research which is freely available for redistribution 